JOURNAL OF THE

AMERICAN MATHEMATICAL SOCIETY

Volume 4, Number 3, July 1991

\title{
THE BOUNDARY OF NEGATIVELY CURVED GROUPS
}

\author{
MLADEN BESTVINA AND GEOFFREY MESS
}

\section{INTRODUCTION}

Every finitely generated group $\Gamma$ can be endowed with the word metric $d$ (invariant under left translations) with respect to a given finite generating set. Following Gromov [Gr] we say that $\Gamma$ is negatively curved provided there exists a (large) number $\delta$ such that

$$
(x \cdot y) \geq \min ((x \cdot z),(y \cdot z))-\delta
$$

for all $x, y, z \in \Gamma$. Here $(x \cdot y)$ stands for the "overlap function"

$$
\frac{1}{2}(d(x, 1)+d(y, 1)-d(x, y)) .
$$

Gromov's article $[\mathrm{Gr}]$ contains fundamental properties of negatively curved groups. Several sets of seminar notes are available [FrN, SwN, USN] that contain more detailed accounts of, and further expand on, Gromov's article.

A sequence $x_{i} \in \Gamma, i=1,2, \ldots$, is convergent at infinity provided $\left(x_{i} \cdot x_{j}\right) \rightarrow \infty$ as $i, j \rightarrow \infty$. Two sequences $\left\{x_{i}\right\},\left\{y_{i}\right\}$ convergent at infinity are equivalent if $\left(x_{i} \cdot y_{i}\right) \rightarrow \infty$ as $i \rightarrow \infty$. A point in the boundary $\partial \Gamma$ of $\Gamma$ is an equivalence class of sequences convergent at infinity. Now $\Gamma \cup \partial \Gamma$ has a natural topology, in which $\Gamma$ is a discrete subspace, and a typical (not necessarily open) neighborhood of a point $a \in \partial \Gamma$ represented by a sequence $\left\{x_{i}\right\}$ is given by $\{y \in \Gamma \cup \partial \Gamma \mid(a \cdot y)>N\}$, where $(a \cdot y)=\lim \left(x_{i} \cdot y\right)$ if $y \in \Gamma$, and $(a \cdot y)=\lim \left(x_{i} \cdot y_{i}\right)$ if $y \in \partial \Gamma$ is represented by a sequence $\left\{y_{i}\right\} . \partial \Gamma$ is a compact, metrizable, finite-dimensional space [Gr, SwN].

The important tool that relates $\partial \Gamma$ with the cohomological properties of $\Gamma$ is the Rips complex $P_{d}(\Gamma)$. For every $d \geq 0, P_{d}(\Gamma)$ is the simplicial complex whose vertices are elements of $\Gamma$, and a collection $x_{1}, \ldots, x_{k} \in \Gamma$ spans a simplex if $d\left(x_{i}, x_{j}\right) \leq d$ for all $i, j$. The natural group action of $\Gamma$ on itself by left translations gives rise to an action on $P_{d}(\Gamma)$. The key observation of Rips is that when $d$ is sufficiently large, $P_{d}(\Gamma)$ is contractible and therefore, when $\Gamma$ is torsion free, provides a model for $E \Gamma$. More precisely, we have

Proposition 1.1 [Gr, Lemma 1.7.A; SwN, §4.2, Proposition 9]. Suppose that $\Gamma$ is a negatively curved group, and let $\delta$ be as in the definition. Choose an integer

Received by the editors June 12, 1990.

1991 Mathematics Subject Classification. Primary 20F32; Secondary 20J05, 57M40. 
$d \geq 4 \delta+2$, and set $P(\Gamma)=P_{d}(\Gamma)$. For every finite simplicial complex $K$, every simplicial map $f: K \rightarrow P(\Gamma)$, and every vertex $v$ of $P(\Gamma)$, there is a homotopy $H: K \times[0,1] \rightarrow P(\Gamma)$ such that

(1) $H_{0}=f$;

(2) $H_{1}=v$;

(3) $H(K \times[0,1])$ is contained in the subcomplex spanned by the vertices of $P(\Gamma)$ which lie on a shortest path joining $v$ and a point of $f\left(K^{(0)}\right)$.

When $\Gamma$ has torsion, we can construct a highly connected polyhedron with free and cocompact $\Gamma$-action. For integers $d$ and $m$, let $P(d, m, \Gamma)$ be the subcomplex of the $m$-fold join $\Gamma * \cdots * \Gamma$ consisting of those simplices whose vertices are within $d$ in $\Gamma$. The natural map $P(d, m, \Gamma) \rightarrow P_{d}(\Gamma)$ has the property that the preimage of each simplex is $(m-2)$-connected. It follows that for large $d, P(d, m, \Gamma)$ is an $(m-2)$-connected free $\Gamma$-complex with compact quotient. This shows for example that $\Gamma$ is a group of type $F P_{\infty}$. Furthermore, for any ring $R$ (in practice $R=\mathbf{Z}$ or $\mathbf{Q}$ ) the compactly supported cohomology $H_{c}^{i}(P(d, m, \Gamma) ; R)$, which is (by definition, see [Br]) isomorphic to $H^{i}(\Gamma ; R \Gamma)$ for $i<m-2$, is also isomorphic to $H_{c}^{i}\left(P_{d}(\Gamma) ; R\right)$ for $i<m-2$. Thus we have that for all $i, H^{i}(\Gamma ; R \Gamma) \cong H_{c}^{i}\left(P_{d}(\Gamma)\right.$ ) (for large $d$ ). From now on, we set $P(\Gamma)$ to be $P_{d}(\Gamma)$ for large $d$.

Let $\overline{P(\Gamma)}$ denote $P(\Gamma) \cup \partial \Gamma$, where a typical neighborhood of a point $a \in \partial \Gamma$ is defined to be a typical neighborhood $U$ of $a$ in $\Gamma \cup \partial \Gamma$, together with the subcomplex of $P(\Gamma)$ spanned by the vertices of $P(\Gamma)$ in $U$. Then $\overline{P(\Gamma)}$ is a compact, metrizable, finite-dimensional [Gr, SwN] space.

Recall [An] that a closed subset $Z$ in a compact ANR $X$ is a $Z$-set (or a set of infinite deficiency) if for every open set $U$ in $X$ the inclusion $U-Z \hookrightarrow U$ is a homotopy equivalence. For example, any closed subset of the boundary of a manifold is a $Z$-set in the manifold. Using standard methods (see, e.g., [He]) one can show that each of the following properties characterizes $Z$-sets:

(1) For every $\varepsilon>0$ there is a map $X \rightarrow X-Z$, which is $\varepsilon$-close to the identity.

(2) For every closed $A \subset Z$ there is a homotopy $H: X \times[0,1] \rightarrow X$ such that $H_{0}=$ identity, $H_{t} \mid A=$ inclusion, and $H_{t}(X-A) \subset X-Z$ for $t>0$.

Theorem 1.2. $\overline{P(\Gamma)}$ is an absolute retract $(A R)$, and $\partial \Gamma \subset \overline{P(\Gamma)}$ is a Z-set.

The proof occupies $\S 2$.

Corollary 1.3. Let $\Gamma$ be a negatively curved group.

(a) $\partial \Gamma$ determines the proper homotopy type of $P(\Gamma)$. More precisely, if $\partial \Gamma$ is embedded as a $Z$-set in a compact $A R R$, then $R-\partial \Gamma \simeq_{p . h . e .} P(\Gamma)$.

(b) For every ring $R$ there is an isomorphism of $R \Gamma$-modules

$$
H^{i}(\Gamma ; R \Gamma) \cong \check{H}^{i-1}(\partial \Gamma ; R) \quad(\check{C} \text { ech, reduced })
$$


(c) $\Gamma$ is a Poincaré duality group of dimension $n$ iff $\Gamma$ is torsion free and $\partial \Gamma$ has the integral Čech cohomology of a sphere of dimension $n-1$.

(d) $\partial \Gamma$ is never cell-like.

Before stating the next corollary, recall that for any (compact, metric) space $X$ and any ring $R$ the cohomological dimension of $X$ over $R$ is defined as

$$
\operatorname{dim}_{R} X=\sup \left\{n \mid \check{H}^{n}(X, A ; R) \neq 0 \text { for some closed set } A \subset X\right\} .
$$

It is known that if the (covering) dimension of $X$ is finite, then $\operatorname{dim} X=$ $\operatorname{dim}_{\mathrm{z}} X$. For a geometric proof of this fact see [Wa]. The classical question, due to Alexandrov, whether this is true for infinite-dimensional $X$, has recently been answered in the negative by Dranišnikov [Dr]. It is clear that for any $X$, $\operatorname{dim}_{\mathrm{Q}} X \leq \operatorname{dim} X$, but there are examples where strict inequality holds. For example, start with a triangulation of the 2-sphere. Remove each 2-simplex and sew a Möbius band instead to obtain a nonorientable surface. Now retriangulate so that the mesh of the new triangulation is much smaller than before and repeat the above process. The limiting space $X$ is two-dimensional (it even has a fundamental class over $\mathbf{Z}_{2}$ ), but $\operatorname{dim}_{\mathbf{Q}} X=1$.

Analogously, for any group $\Gamma$ and a ring $R$, the cohomological dimension of $\Gamma$ over $R$ is defined as

$$
\operatorname{cd}_{R} \Gamma=\sup \left\{n \mid H^{n}(\Gamma ; M) \neq 0 \text { for some } R \Gamma \text {-module } M\right\} .
$$

When $\operatorname{cd}_{R} \Gamma$ is finite and $\Gamma$ is $F P_{\infty}, \operatorname{cd}_{R} \Gamma=\max \left\{n \mid H^{n}(\Gamma ; R \Gamma) \neq 0\right\}$. This of course fails when $\operatorname{cd}_{R} \Gamma$ is infinite, as can be seen by taking $R=\mathbf{Z}$ and $\Gamma$ a finite group. The geometric dimension of $\Gamma$ is the minimal dimension of a $K(\Gamma, 1)$-complex. It is known that the geometric dimension equals $\mathrm{cd}_{\mathbf{z}}$ except possibly in one case (which is still unknown). For a discussion of this see [Br]. If $\Gamma$ contains a torsion-free subgroup of finite index, its virtual cohomological dimension vcd is defined to be the integral cohomological dimension (denoted $\mathrm{cd}$ in the sequel) of any such subgroup.

Using the technique of Gromov and Davis-Januszkiewicz, it is possible to construct a negatively curved group $\Gamma$ whose boundary is the space mentioned above. Let $P$ be a polyhedron that is a 3 -manifold in the complement of a single vertex, and so that the link of that vertex is a nonorientable surface. Then as in [DJ], hyperbolize $P$ preserving this property, and let $\Gamma$ be its fundamental group. Arguments in [DJ] show that the boundary of the universal cover of $P$ is two-dimensional, but its rational cohomological dimension is one; analogously, the integral cohomological dimension of $\Gamma$ is three, while its rational cohomological dimension is two.

Corollary 1.4. Let $\Gamma$ be a negatively curved group, and let $R$ be a ring with 1 .

(a) $\operatorname{dim}_{R} \partial \Gamma=\max \left\{n \mid H^{n}(\Gamma ; R \Gamma) \neq 0\right\}$.

(b) $\operatorname{dim} \partial \Gamma=\max \left\{n \mid H^{n}(\Gamma ; \mathbf{Z} \Gamma) \neq 0\right\}$.

(c) If $\operatorname{cd}_{R} \Gamma<\infty$ then $\operatorname{dim}_{R} \partial \Gamma=\operatorname{cd}_{R} \Gamma-1$.

(d) If $\Gamma$ is torsion free then $\operatorname{dim} \partial \Gamma=\operatorname{cd} \Gamma-1$. 
(e) If $\Gamma$ is virtually torsion free (e.g., if it is residually finite) then $\operatorname{dim} \partial \Gamma=$ $\operatorname{vcd} \Gamma-1$.

In $\S 3$ we discuss the local connectivity of $\partial \Gamma$. In $\S 4$ we consider closed, irreducible 3-manifolds with infinite negatively curved fundamental group. Both Valentin Poenaru [Po] and Andrew Casson [C] show that (even under a weaker hypothesis on the fundamental group) universal covers of such manifolds are homeomorphic to $\mathbf{R}^{3}$. We make this result more precise by showing that the universal covers compactified by $\partial \Gamma$ are homeomorphic to the 3-ball. According to Thurston's Geometrization Conjecture, these manifolds should support a metric of constant negative sectional curvature.

\section{Proof of Theorem 1.2}

Proposition 2.1. Suppose that $X$ is a compactum and $Z \subset X$ a closed subset such that

(i) int $Z=\varnothing$;

(ii) $\operatorname{dim} X=n<\infty$;

(iii) for every $k=0,1, \ldots, n$, every point $z \in Z$, and every neighborhood $U$ of $z$, there is a neighborhood $V$ of $z$ such that every map $\alpha: S^{k} \rightarrow V-Z$ extends to $\tilde{\alpha}: B^{k+1} \rightarrow U-Z$;

(iv) $X-Z$ is an $A N R$.

Then $X$ is an $A N R$ and $Z \subset X$ is a $Z$-set.

The proof of the proposition is based on standard techniques (see, e.g., [Bo]). Recall that a map $f$ defined on a subcomplex $\operatorname{Dom}(f)$ of a simplicial complex $K$ that contains all vertices with values in a space $Y$ with an open cover $\mathscr{U}$ is said to be a $\mathscr{U}$-partial realization if for every simplex $\sigma \subset K$ the set $f(\sigma \cap \operatorname{Dom}(f))$ is $\mathscr{U}$-small. A $\mathscr{U}$-partial realization $f$ is full if $f$ is defined on all of $K$.

Lemma 2.2. For every open cover $\mathscr{U}$ of $X$ there is an open cover $\mathscr{V}$ of $X$ such that every $\mathscr{V}$-partial realization of an $(n+1)$-complex into $X-Z$ extends to a full $\mathscr{U}$-realization into $X-Z$.

Proof. Let $\mathscr{U}=\mathscr{U}_{n+1}>\mathscr{U}_{n}>\cdots>\mathscr{U}_{1}>\mathscr{U}_{0}$ be a sequence of open covers of $X$ so that for every element $U^{\prime}$ of $\mathscr{U}_{i}$ there exists an element $U^{\prime \prime}$ of $\mathscr{U}_{i+1}$ such that every $S^{i} \rightarrow U^{\prime}-Z$ extends to $B^{i+1} \rightarrow U^{\prime \prime}-Z \quad(i=0,1, \ldots, n)$. Set $\mathscr{V}=\mathscr{U}_{0}$, and let $f=f_{0}$ be a partial $\mathscr{V}$-realization into $X-Z$ defined on a subcomplex of an $(n+1)$-complex $K$. Inductively extend $f$ to a partial $\mathscr{U}_{i}$ realization $f_{i}$ defined on the original subcomplex union the $i$-skeleton. Then $f_{n+1}$ is the desired extension.

Lemma 2.3. For every open cover $\mathscr{U}$ of $X$ and every map $f: P \rightarrow X$ of an $(n+1)$-dimensional polyhedron, there is a map $g: P \rightarrow X-Z$ U-close to $f$.

Proof. Let $\mathscr{U}^{\prime}$ be a star-refinement of $\mathscr{U}, \mathscr{V}$ as in Lemma 2.2 with respect to $\mathscr{U}^{\prime}$, and let $\mathscr{W}$ be such that $\operatorname{St}^{2}\left(\mathscr{U}^{\prime}, \mathscr{W}\right)<\mathscr{U}, \operatorname{St}(\mathscr{W}, \mathscr{W})<\mathscr{V}$. Triangulate 
$P$ so that each simplex is sent by $f$ into an element of $\mathscr{W}$. Define $g$ on $P^{(0)}$ (using the fact that $Z$ is nowhere dense) so that $g\left(P^{(0)}\right) \subseteq X-Z$ and $f$ and $g$ are $\mathscr{W}$-close on $P^{(0)}$.

Then $g$ is a partial $\mathscr{V}$-realization, hence it extends to a full $\mathscr{U}^{\prime}$-realization $g: P \rightarrow X-Z$. Now $g$ and $f$ are $\mathscr{U}$-close.

Lemma 2.4. For every $f: P \rightarrow X$, where $P$ is a polyhedron of dimension $\leq n$, there is a homotopy $H: P \times[0,1] \rightarrow X$ so that $H_{0}=f$ and $H(P \times(0,1]) \subset$ $X-Z$.

Proof. Let $\mathscr{U}_{1}, \mathscr{U}_{2}, \ldots$ be a sequence of open covers of $X$ with mesh $\mathscr{U}_{i} \rightarrow 0$. Let $\mathscr{V}_{i}$ be an open cover of $X$ so that $\mathrm{St} \mathscr{V}_{i}$ is as in Lemma 2.2 with respect to $\mathscr{U}_{i}$.

Define $H$ on $P \times\{1 / n\}$ using Lemma 2.3 so that $H_{1 / n}$ is $\mathscr{V}_{n}$-close to $f$, $H_{1 / n}(P) \subset X-Z$. Since $H_{1 / n}$ and $H_{1 / n+1}$ are $\mathrm{St} \mathscr{V}_{n}$-close, we can extend $H$ to $P \times[1 /(n+1), 1 / n]$ so that $H(\mathrm{pt} \times[1 /(n+1), 1 / n])$ is $\mathscr{U}_{n}$-small $(n=$ $1,2,3, \ldots)$.

Lemma 2.5. For every $z \in Z$, every $k=0,1, \ldots, n$, and every neighborhood $U$ of $z$, there is a neighborhood $V$ of $z$ such that every $f: S^{k} \rightarrow V$ extends to $\tilde{f}: B^{k+1} \rightarrow U$.

Proof. Let $V$ be as in the hypothesis of Proposition 2.1. By Lemma 2.4 there is $H: S^{k} \times[0,1] \rightarrow X$ with $H_{0}=f, H\left(S^{k} \times(0,1]\right) \subset X-Z$. For some $\varepsilon>0, H\left(S^{k} \times[0, \varepsilon]\right) \subset V$. Now $H_{\varepsilon}$ extends to $B^{k+1}$ by assumption.

Proof of Proposition 2.1. $X$ is $n$-dimensional and locally $n$-connected (by Lemma 2.5) hence an ANR. Lemma 2.3 now implies that $Z$ is a $Z$-set.

Proof of Theorem 1.2. Apply Proposition 2.1 to $X=\overline{P(\Gamma)}, Z=\partial \Gamma$. Then (iv) is obvious and (i) and (ii) follow from [Gr]. To verify property (iii), assume $U$ is a neighborhood of $z \in \partial \Gamma$. There is $R>0$ such that $U$ contains the closure of the subcomplex of $P(\Gamma)$ spanned by vertices $w$ of $P(\Gamma)$ such that $(z \cdot w) \geq R$. Define $V$ to be the closure of the subcomplex of $P(\Gamma)$ spanned by vertices $w$ of $P(\Gamma)$ with $(z \cdot w) \geq 2 R+3 \delta$.

Now assume that $w_{1}, w_{2}, w$ are vertices in $P(\Gamma)$ with $d\left(w_{1}, w\right)+d\left(w, w_{2}\right)$ $=d\left(w_{1}, w_{2}\right)$ and $w_{1}, w_{2} \in V$. We claim that $w \in U$. First note that $\left(w_{1}\right.$. $\left.w_{2}\right) \geq \min \left((w, z),\left(w_{2}, z\right)\right)-\delta \geq 2 R+2 \delta$ and that $\left(w_{1} \cdot w\right)+\left(w_{2} \cdot w\right)=$ $\left(w_{1} \cdot w_{2}\right)+d(w, 1) \geq 2 R+2 \delta$. In particular, $\left(w_{i} \cdot w\right) \geq R+\delta$ for $i=1$ or 2 . But then $(z \cdot w) \geq \min \left(\left(z \cdot w_{i}\right),\left(w_{i} \cdot w\right)\right)-\delta \geq R$, hence $w \in U$. Proposition 1.1 now implies that every finite subcomplex of $V$ is contractible inside $U$.

Proposition 2.1 now implies that $\partial \Gamma$ is a $Z$-set in $\overline{P(\Gamma)}$. Therefore inclusion $P(\Gamma) \rightarrow \overline{P(\Gamma)}$ is a homotopy equivalence, so $\overline{P(\Gamma)}$ is contractible. But contractible ANR's are AR's.

Proof of Corollary 1.3. (a) The identity $\partial \Gamma \rightarrow \partial \Gamma$ extends to a map $\alpha: R \rightarrow$ $\overline{P(\Gamma)}$ with $\alpha(R-\partial \Gamma) \subseteq P(\Gamma)$, and similarly to a map $\beta: \overline{P(\Gamma)} \rightarrow R$ with 
$\beta(P(\Gamma)) \subseteq R-\partial \Gamma$, because $\partial \Gamma$ is a $Z$-set in both spaces. Then the restrictions of $\alpha$ and $\beta$ are proper homotopy equivalences between $R-\partial \Gamma$ and $P(\Gamma)$, and they are each others' proper homotopy inverses. We can make this even more precise. Recall that the Chapman Complement Theorem [Ch] states that two $Z$-sets in the Hilbert cube $I^{\infty}$ have the same shape if and only if their complements are homeomorphic. It follows that the shape of $\partial \Gamma$ determines the homeomorphism type of $P(\Gamma) \times I^{\infty}$ and vice versa.

(b) This follows from the cohomology exact sequence with coefficients in $R$ for the pair $(\overline{P(\Gamma)}, \partial \Gamma)$, and $\breve{H}^{i}(\overline{P(\Gamma)}, \partial \Gamma ; R) \cong H_{c}^{i}(P(\Gamma) ; R) \cong H^{i}(\Gamma, R \Gamma)$.

(c) Follows from (b).

(d) If $n=\operatorname{cd}_{\mathbf{Q}} \Gamma<\infty$, then $[\mathrm{Br}] 0 \neq H^{n}(\Gamma ; \mathbf{Q} \Gamma) \cong \check{H}^{n-1}(\partial \Gamma$; $\mathbf{Q})$.

To motivate the proof of Corollary 1.4 we first prove

Proposition 2.6. Suppose $X$ is a finite-dimensional $A N R$ and $Z \subset X$ a $Z$-set. Then $\operatorname{dim} Z<\operatorname{dim} X$ (and hence $\operatorname{dim} Z<\operatorname{dim}(X-Z)$ ).

Proof. Suppose $\operatorname{dim} X=\operatorname{dim} Z=n<\infty$. Then there is a closed set $A \subset Z$ such that $\breve{H}^{n}(Z, A) \neq 0$. Let $H: X \times[0,1] \rightarrow X$ be a homotopy such that (i) $H_{0}=$ identity, (ii) $H_{t} \mid A=$ inclusion, (iii) $H_{t}(X-A) \subset(X-Z)$ for $t>0$. Set $B_{2}=H_{1}(X), B_{1}=H\left(B_{2} \times[0,1]\right)$. Then $X \supset B_{1} \supset B_{2}, B_{1} \cap Z=B_{2} \cap Z=A$. Also, $\breve{H}^{n}\left(X, B_{1}\right) \rightarrow \breve{H}^{n}\left(X, B_{2}\right)$ is 0 . (All cohomology groups are Čech and integral.)

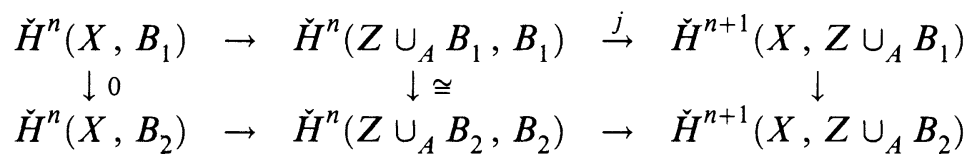

Groups in the middle column are isomorphic to $\check{H}^{n}(Z, A) \neq 0$. A diagram chase shows that $j$ is $1-1$ and hence $\check{H}^{n+1}\left(X, Z \cup B_{1}\right) \neq 0$, contradicting $\operatorname{dim} X=n$.

Proof of Corollary 1.4. (a) Put $k=\max \left\{n \mid H^{n}(\Gamma ; R \Gamma) \neq 0\right\}$ and $m=$ $\operatorname{dim} P(\Gamma)$. Then by $1.3(\mathrm{~b})$ we have $\breve{H}^{k-1}(\partial \Gamma ; R) \cong H^{k}(\Gamma ; R \Gamma) \neq 0$ and, therefore, $\operatorname{dim}_{R} \partial \Gamma \geq k-1$. In case $m=k$ the reverse inequality follows from Proposition 2.6. In general, we argue as follows.

By $C^{i}$ denote the free $R$-module whose basis consists of $i$-simplices $\sigma^{i}$ of $P(\Gamma)$. Then $C^{i}$ is a finitely generated $R \Gamma$-module, in general not free unless $\Gamma$ is torsion free. The elements of $C^{i}$ are $i$-cochains in $P(\Gamma)$ with coefficients in $R$ and compact support. We denote the coboundary $C^{i} \rightarrow C^{i+1}$ by $\delta$. We now claim that there exists an integer $K$ such that every cocycle $z \in C^{i}$ $(i>k)$ is the coboundary of a cochain whose support is contained in the combinatorial $K$-neighborhood of the support of $z$. To see this, we construct $R$-homomorphisms $\Delta^{i}: C^{i} \rightarrow C^{i-1}$ for $m \geq i>k$ so that $\delta \Delta^{i}+\Delta^{i+1} \delta=$ id (a cochain homotopy from id to 0 above dimension $k$ ) as follows. First define $\Delta^{m}$ on a representative $\sigma$ of each $\Gamma$-equivalence class of $m$-simplices in $P(\Gamma)$ (viewed as $m$-cocycles with weight $1 \in R$ ) to be an arbitrary cochain in $C^{m-1}$ 
cobounding $\sigma$. (Here we use the fact that $H_{c}^{m}(P(\Gamma) ; R)=0$.) Then for any other $m$-simplex $\sigma$ choose an element $\gamma \in \Gamma$ such that $\gamma(\sigma)$ is one of the chosen representatives, and then define $\Delta^{m}(\sigma)$ to be the $\gamma^{-1}$-translate of $\Delta^{m}(\gamma(\sigma))$. If $\Gamma$ is not torsion free, then $\gamma$ is not unique and $\Delta^{m}$ is not $\Gamma$ equivariant, but in any case there is a constant $K_{m}$ such that any $m$-cocycle $z$ in $C^{m}$ is the coboundary of the cochain $\Delta^{m}(z)$ whose support is contained in the $K_{m}$-neighborhood of the support of $z$. Furthermore, for each $(m-1)$ simplex $\sigma$ consider the cocycle (and hence a coboundary) $A_{\sigma}=\sigma-\Delta^{m} \delta(\sigma)$. Say that two $(m-1)$-simplices $\sigma_{1}$ and $\sigma_{2}$ are equivalent if there is $\gamma \in \Gamma$ with $\gamma\left(\sigma_{1}\right)=\sigma_{2}$ and $\gamma\left(A_{\sigma_{1}}\right)=A_{\sigma_{2}}$. There are only finitely many equivalence classes of $(m-1)$-simplices. Now inductively assume that $\Delta^{i+1}$ is constructed, that $i>k$, and that there are only finitely many equivalence classes of $i$-simplices $\sigma$ with respect to the equivalence relation $\sigma_{1} \sim \sigma_{2}$ iff there is $\gamma \in \Gamma$ such that $\gamma\left(\sigma_{1}\right)=\sigma_{2}$ and $\gamma\left(A_{\sigma_{1}}\right)=A_{\sigma_{2}}$, where $A_{\sigma}=\sigma-\Delta^{i+1} \delta(\sigma)$. Define $\Delta^{i}$ on a representative $\sigma$ of an equivalence class of $i$-simplices in $P(\Gamma)$ to be an arbitrary cochain in $C^{i-1}$ cobounding $A_{\sigma}$ (which is a cocycle and thus a coboundary) and then extend to all $i$-simplices as before. This completes the proof of the claim.

Assuming $\operatorname{dim}_{R} \partial \Gamma=i \geq k$, let $A$ be a closed subset of $\partial \Gamma$ with

$$
H^{i}(\partial \Gamma, A ; R) \neq 0,
$$

and construct closed sets $\partial \Gamma=X \supset B_{0} \supset B_{1} \supset B_{2}$ as in the proof of Proposition 2.6 such that

(i) homomorphisms $\check{H}^{i}\left(X, B_{j} ; R\right) \rightarrow \check{H}^{i}\left(X, B_{j+1} ; R\right)$ are 0 for $j=0,1$;

(ii) $B_{j} \cap \partial \Gamma=A$ for $j=0,1,2$; and

(iii) each $B_{j} \cap P(\Gamma)$ is a subcomplex, and all cells in $P(\Gamma)$ at distance $\leq K$ from $B_{1} \cap P(\Gamma)$ are contained in $B_{0}$, so that in particular the homomorphism $H_{c}^{i+1}\left(P(\Gamma), B_{0} \cap P(\Gamma) ; R\right) \rightarrow H_{c}^{i+1}\left(P(\Gamma), B_{1} \cap P(\Gamma) ; R\right)$ induced by inclusion is 0 .

Arguing as in Proposition 2.6, we see that the homomorphism

$$
\check{H}^{i+1}\left(X, \partial \Gamma \cup_{A} B_{0} ; R\right) \rightarrow \check{H}^{i+1}\left(X, \partial \Gamma \cup_{A} B_{1} ; R\right)
$$

is nonzero, because both groups have to carry $\check{H}^{i}(\partial \Gamma, A ; R)$, thus contradicting (iii), because $H_{c}^{i+1}\left(P(\Gamma), B_{j} \cap P(\Gamma) ; R\right) \cong \check{H}^{i+1}\left(X, \partial \Gamma \cup_{A} B_{j} ; R\right)$.

(b) Follows from (a) for $R=\mathbf{Z}$ and the fact that $\operatorname{dim}_{\mathbf{Z}} X=\operatorname{dim} X$, provided the latter is finite.

(c) Follows from (a) and the fact that $\max \left\{n \mid H^{n}(\Gamma ; R \Gamma) \neq 0\right\}=\mathrm{cd}_{R} \Gamma$, assuming the latter is finite.

(d) Follows from (b) and the fact that a torsion-free negatively curved group has finite integral cohomological dimension.

(e) Follows from (c) applied to a torsion-free subgroup of finite index, and the fact that such a subgroup has the same boundary as $\Gamma$. 


\section{LOCAL CONNECTIVITY OF THE BOUNDARY}

In this section we assume that $\Gamma$ is a one-ended negatively curved group, or equivalently that $\partial \Gamma$ is connected. It was first conjectured by G. Mess and M. Mihalik that in this case $\partial \Gamma$ is locally connected. Ross Geoghegan has pointed out that Theorem 1.2 implies that $\Gamma$ is semistable at infinity if and only if $\partial \Gamma$ has the shape of a locally connected continuum. (For a proof, see [DS, 7.2.3].)

Before stating the results of this section, recall that $P(\Gamma)$ (as well as each negatively curved space) admits a constant $\delta^{\prime}$ such that every point on one of the sides of a geodesic triangle is $\delta^{\prime}$-close to the union of the other two sides. An infinite geodesic ray is a map $r:[0, \infty) \rightarrow P(\Gamma)$ such that $d(r(a), r(b))=$ $|a-b|$. Every such ray converges to a point $r(\infty) \in \partial \Gamma$. We are grateful to Mike Mihalik for pointing out the following fact:

Lemma 3.1. There is a constant $C$ such that for every $x \in P(\Gamma)$ there exists an infinite geodesic ray starting at 1 which passes within $C$ of $x$.

Proof. It is well known that there are bi-infinite geodesics in $P(\Gamma)$. By translating by an element of the group, we see that there is a bi-infinite geodesic $g$ passing close to $x$. Then one of the two rays joining 1 with the endpoints of $g$ will pass close to $x$.

Consider the following property that $P(\Gamma)$ may or may not satisfy for a number $M>0$.

$\left(\ddagger_{M}\right) \quad$ There is a number $L>0$ such that for every $R>0$ and any two points $x, y \in P(\Gamma)$ with $d(1, x)=d(1, y)=R$ and $d(x, y) \leq M$, there exists a path of length $\leq L$ connecting $x$ to $y$ in the complement of the ball of radius $R-C$ centered at 1 .

(Note that since $\Gamma$ has one end, Lemma 3.1. implies that $x$ and $y$ are connected by some path missing the ball.)

Proposition 3.2. Suppose that $P(\Gamma)$ satisfies $\left(\ddagger_{M}\right)$ for some $M \geq 6 C+2 \delta^{\prime}+3$. Then $\partial \Gamma$ is locally connected (and hence $\Gamma$ is semistable at infinity).

Proof. We may assume that the number $L$ in $\left(\ddagger_{M}\right)$ is an integer. Let $x$ and $y$ be two points in $\partial \Gamma$. We construct a map $\varphi:[0,1] \rightarrow \partial \Gamma$ such that $\varphi(0)=x$, $\varphi(1)=y$. Furthermore, if $x$ and $y$ are close, the image of $\varphi$ will be small.

Let $r_{0}$ and $r_{1}$ be geodesic rays connecting 1 to $x$ and $y$. By induction on $k=1,2, \ldots$ we construct geodesic rays $r_{i / L^{k}}$, where $i$ is a positive integer smaller than $L^{k}$ and not divisible by $L$, satisfying

$$
d\left(r_{i / L^{k}}(k), r_{i+1 / L^{k}}(k)\right) \leq 6 C+2 \delta^{\prime}+3 \text {. }
$$

Inductive step. By $\left(\ddagger_{M}\right)$ there is a path $\alpha:[0, L] \rightarrow P(\Gamma)$ parametrized with speed $\leq 1$ joining $r_{i / L^{k}}(k)$ to $r_{i+1 / L^{k}}(k)$ in the complement of the ball of radius $k-C$. Interpolate the required $L-1$ infinite geodesic rays so that they 
pass through points $y_{1}, y_{2}, \ldots, y_{L-1}$ within $C$ of $\alpha(1), \alpha(2), \ldots, \alpha(L-1)$. Then the $y$ 's are at distance $\geq k-2 C$ from 1 . Find points $z_{1}, z_{2}, \ldots, z_{L-1}$ on these rays within $2 C+1$ of the $y$ 's that are at distance at least $k+1$ from 1. The consecutive $z$ 's are within $6 C+3$. Now a look at the geodesic triangle $\Delta 1 z_{j} z_{j+1}$ reveals the inductive statement for $k+1$. Define $\varphi\left(i / L^{k}\right)$ to be the endpoint of $r_{i / L^{k}}$. Clearly, $\varphi$ extends continuously to $[0,1]$.

We now turn to the study of $\partial \Gamma$ in case $P(\Gamma)$ fails to satisfy $\left(\ddagger_{M}\right)$.

Proposition 3.3. Suppose that $\left(\ddagger_{M}\right)$ fails for some $M>0$. Then $\partial \Gamma$ contains a cut (= separating) point.

Proof. We take a sequence of worse and worse examples. Let $x_{n}, y_{n}$ be points at distance $R_{n}$ from 1 and $\leq M$ from each other, so that there is no path of length $\leq n$ joining $x_{n}$ to $y_{n}$ in the complement of the ball $B_{n}$ of radius $R_{n}-C$ centered at 1 . Let $r_{n}, s_{n}$ be infinite geodesic rays from 1 passing within $C$ of $x_{n}, y_{n}$ respectively. Parametrize them by $\left[-R_{n}, \infty\right)$ and note that $d\left(x_{n}, r_{n}(0)\right) \leq 2 C, d\left(y_{n}, s_{n}(0)\right) \leq 2 C$. After possibly passing to a subsequence, we can find $\gamma_{n} \in \Gamma$ such that: $\gamma_{n}\left(x_{n}\right) \rightarrow x \in P(\Gamma), \gamma_{n}\left(y_{n}\right) \rightarrow y \in P(\Gamma)$, $\gamma_{n}(1) \rightarrow c \in \partial \Gamma, \gamma_{n}\left(B_{n}\right) \rightarrow B \subset \overline{P(\Gamma)}$ (in the Hausdorff topology; think of $B$ as a "horoball"), $\gamma_{n}\left(r_{n}\right) \rightarrow r$, and $\gamma_{n}\left(s_{n}\right) \rightarrow s$, where $r, s$ are biinfinite geodesics from $c$ to $a, b \in \partial \Gamma$ respectively. We now claim that $B \cap \partial \Gamma=\{c\}$. It is clear that $c \in B$. For every $d \in \partial \Gamma-\{c\}$ there is $N>0$ such that every geodesic joining points $e, f$ close enough to $c, d$ respectively, passes within $N$ of $x$ ("there are no shortcuts in a negatively curved space"). Apply this to $e=\gamma_{n}(1)$ to get $d\left(\gamma_{n}(1), f\right) \geq R_{n}-N+d(x, f)>R_{n}$ for $d(x, f)>N$.

We show that $a$ and $b$ belong to distinct components of $\partial \Gamma-\{c\}$. Choose an open cover $\mathscr{U}$ of $\partial \Gamma-\{c\}$ such that if $p_{i} \in U_{i} \in \mathscr{U}$ for $i=1,2$ and if $U_{1} \cap U_{2} \neq \varnothing$, then any geodesic connecting $p_{1}$ to $p_{2}$ misses $B$. Any chain $a \in U_{1}, U_{2}, \ldots, U_{k} \ni b$ of elements of $\mathscr{U}$ with $U_{i} \cap U_{i+1} \neq \varnothing$ would give rise to a path connecting $a$ to $b$ in $\overline{P(\Gamma)}-B$. This path, gently pushed into $P(\Gamma)$, together with segments of geodesics $r$ and $s$, and geodesics joining $x$ to $r(0)$ and $y$ to $s(0)$, gives us a path $\alpha$ joining $x$ to $y$, which is disjoint from $B$ except for two parts of length $\leq 2 C$ adjacent to the endpoints. For large $n$, the path $\gamma_{n}^{-1}(\alpha)$ contradicts the choice of $x_{n}, y_{n}$.

Proposition 3.4. Assume that $\partial \Gamma$ contains a cut point, and let $R$ be a principal ideal domain. Then every nonzero $R$-module $\check{H}^{i}(\partial \Gamma ; R)$ for $i>0$ is infinitely generated (= not finitely generated).

Proof. Represent $\partial \Gamma=A \cup B$ with $A \cap B=\{c\}$ and so that $A$ and $B$ are closed and have nonempty interior. Because $\check{H}^{i}(\partial \Gamma ; R)=\check{H}^{i}(A ; R) \oplus \check{H}^{i}(B ; R)$, we can assume that $\check{H}^{i}(A ; R) \neq 0$. We now argue that for any $k>0$ the direct sum of $k$ copies of $\check{H}^{i}(A ; R)$ is a submodule (even a direct summand) of $\check{H}^{i}(\partial \Gamma ; R)$. This will follow if we can find a collection $c_{1}, c_{2}, \ldots, c_{k}$ of cut points in $\partial \Gamma$ such that $\partial \Gamma=A_{1} \cup A_{2} \cup \cdots \cup A_{k} \cup C_{k}$ for some closed 
subsets $A_{1}, A_{2}, \ldots, A_{k}, C_{k}$ with nonempty interior, $A_{i} \cap A_{j}=\varnothing$ for $i \neq j$, $A_{i} \cap C_{k}=\left\{c_{i}\right\}$, and each $A_{i}$ is homeomorphic to $A$. We prove this by induction on $k$. For $k=1$ we can take $c_{1}=c, A_{1}=A$, and $C_{1}=B$. For the inductive step, suppose that $\partial \Gamma=A_{1} \cup A_{2} \cup \cdots \cup A_{k} \cup C_{k}$ as above. Let $\gamma \in \Gamma$ be an element that fixes points $a, b \in$ int $C_{k}$ [Gr]. Replacing $\gamma$ by a large power if necessary, we may assume that $\gamma\left(A_{1}\right) \subset \operatorname{int} C_{k}$. Now let $A_{k+1}=\gamma\left(A_{1}\right), c_{k+1}=\gamma\left(c_{1}\right)$, and $C_{k+1}=C_{k}-$ int $A_{k+1}$.

Remark. The same argument shows that if a cell-like subset separates $\partial \Gamma$, then every nonzero $R$-module $\breve{H}^{i}(\partial \Gamma ; R)$ for $i>0$ is infinitely generated.

Propositions 3.2-3.4 combined with Corollary 1.3 yield

Theorem 3.5. Let $\Gamma$ be a one-ended negatively curved group and $i>0$ such that $H^{i}(\Gamma ; R \Gamma)$ is a nonzero finitely generated $R$-module for some principal ideal domain $R$. Then $\partial \Gamma$ is locally connected.

\section{3-MANIFOLDS}

This entire section is devoted to a proof of

Theorem 4.1. Let $M$ be a closed, irreducible 3-manifold with negatively curved infinite fundamental group $\Gamma$. Then the universal cover $\widetilde{M}$ of $M$ is homeomorphic to $\mathbf{R}^{3}$ and $\partial \Gamma$ is homeomorphic to the 2-sphere. Furthermore, $B=\widetilde{M} \cup \partial \Gamma$ is homeomorphic to the 3-ball.

By passing to the orientation double cover, we may assume that $M$ is orientable. We first show that $\partial \Gamma$ is a 2 -sphere. Recall the following theorem of Zippin [Wi]: Suppose a compact, metric space $X$ is connected, locally connected, contains more than one point, every embedded circle separates $X$, and no arc in $X$ separates $X$. Then $X$ is homeomorphic to the 2-sphere. We know that $\Gamma$ is torsion free and $H^{i}(\Gamma ; \mathbf{Z} \Gamma) \cong \mathbf{Z}$ for $i=3$ and is trivial otherwise. Corollary 1.3(b) implies that $\partial \Gamma$ is connected and contains more than one point, and Theorem 3.5 asserts that it is locally connected. The remark following Proposition 3.4 shows that no arc in $\partial \Gamma$ separates. We now show that every embedded circle $S$ in $\partial \Gamma$ separates $\partial \Gamma$.

$S$ bounds a 'Čech surface,' that is, there is a compact set $F \subset B=\widetilde{M} \cup \partial \Gamma$ such that

(1) $F \cap \partial \Gamma=S$;

(2) $F \cap \widetilde{M}$ is a properly embedded surface with one end; and

(3) for every compact subsurface $K \subset F \cap \widetilde{M}$ with connected boundary, $S$ and $\partial K$ (with appropriate orientations) are homologous in $F-$ int $K$.

$F$ separates $B$ into two components $U$ and $V$. It is clear that $\partial \Gamma-S=$ $(\partial \Gamma \cap U) \cup(\partial \Gamma \cap V)$, so it suffices to show that $\partial \Gamma \cap U \neq \varnothing$ and $\partial \Gamma \cap V \neq \varnothing$. Choose a neighborhood $N$ of $S$ in $B$ such that $S$ is not null-homologous in $N$. Assuming $\partial \Gamma \cap V=\varnothing$, we conclude that for a sufficiently large compact set $C \subset \widetilde{M}, C \cup N \cup U=B$. By enlarging $C$, we can assume that $C$ is 
a submanifold of $\widetilde{M}$ and it intersects $F$ transversely in a compact surface $K$ with connected boundary. Since $F-\operatorname{int} K \subset N$, we conclude that $S$ is homologous to $\partial K$ in $N$. But $\partial C-U$ is a compact surface in $N$, showing that $\partial K$ is null-homologous in $N$-thus contradicting the choice of $N$. The theorem of Zippin now implies that $\partial \Gamma$ is homeomorphic to the 2-sphere.

Since a neighborhood of $\partial \Gamma$ in $B$ retracts to $\partial \Gamma, \widetilde{M}$ is simply-connected at infinity and therefore homeomorphic to $\mathbf{R}^{3}$. (This also follows from [BT] using only local connectivity of $\partial \Gamma$.)

Finally, we indicate the proof that $B$ is a 3-ball. In [Fe] Steve Ferry proves the following theorem:

(*) $\quad$ Suppose that $X$ is an $A R$ and $Z \subset X$ a $Z$-set such that $Z$ is homeomorphic to $S^{n-1}$ and $X-Z$ is homeomorphic to $\mathbf{R}^{n}$. If $n \geq 5$ then $X$ is homeomorphic to the $n$-ball.

As shown in $[\mathrm{Fe}]$, this theorem is a formal consequence of another theorem in [Fe]: For every open cover $\alpha$ of $\mathbf{R}^{n}(n \geq 5)$ there is an open cover $\beta$ of $\mathbf{R}^{n}$ such that if $g: \mathbf{R}^{n} \rightarrow \mathbf{R}^{n}$ is a map with $\beta$-small point preimages, then $g$ is $\alpha$-homotopic to a homeomorphism. Ferry derives this theorem from the $\alpha$ Approximation Theorem [ChFe]: Let $N^{n}$ be an $n$-manifold $(n \geq 5)$. For every open cover $\alpha$ of $N$ there is an open cover $\beta$ of $N$ such that any $\beta$-equivalence $f: M^{n} \rightarrow N^{n}$ is $\alpha$-close to a homeomorphism. ( $f$ is a $\beta$-equivalence if there is $g: N \rightarrow M$ such that $f g$ is $\beta$-homotopic to the identity.)

Jakobsche [Ja] proved the $\alpha$-Approximation Theorem for $n=3$, assuming additionally that $M$ and $N$ do not contain any fake cells. It now follows by Ferry's argument that $(*)$ holds for $n=3$.

Remark. Theorem 3.1 fails in higher dimensions. Mike Davis and Tadeusz Januszkiewicz [DJ] have examples of aspherical closed manifolds with negatively curved fundamental groups whose universal covers are not homeomorphic to Euclidean space, as well as examples of such manifolds whose universal covers are homeomorphic to Euclidean space, but the boundary fails to be a sphere. However, as a consequence of Ferry's work cited above, if $M$ is an aspherical $n$-manifold $(n \geq 5)$ with negatively curved fundamental group $\Gamma$, and if $\partial \Gamma$ is homeomorphic to the $(n-1)$-sphere, then the universal cover of $M$ compactified by $\partial \Gamma$ is homeomorphic to the $n$-ball.

\section{Questions}

We end the paper with some (hopefully interesting) questions.

Q1. Suppose $\Gamma$ is a group that admits a finite $K(\Gamma, 1)$. When is it possible to compactify the universal cover of this complex equivariantly so that the compactification is an AR and the added set is a $Z$-set? The nonequivariant version of this is discussed in [ChS].

Q2. If $\Gamma$ is a one-ended negatively curved group, is $\partial \Gamma$ necessarily locally connected? Is the condition $(\ddagger)_{M}$ always satisfied? 
Q3. Can the boundary of a one-ended negatively curved group contain a cut point?

Q4. To what extent is the following slogan valid: " $\partial \Gamma$ is the simplest compactum among all compacta of that shape"?, e.g., if $\partial \Gamma$ has the shape of a locally connected continuum, is it locally connected? In one of the Davis-Januszkiewicz examples mentioned above, the boundary has the shape of a sphere (indeed it is a cell-like image of a sphere), but fails to be homeomorphic to it.

\section{ACKNOWLEDGMENTS}

We would like to thank Mike Mihalik for several helpful conversations. Thanks to Bob Edwards and Steve Ferry for their help with Theorem 4.1. The first author acknowledges the support of the Presidential Young Investigator Award and the Alfred P. Sloan Foundation. The second author wishes to thank the Mathematical Sciences Research Institute, I.H.E.S., and the Institute for Advanced Study for their hospitality and support. Both of us acknowledge the support of NSF.

\section{BIBLIOGRAPHY}

[An] R. D. Anderson, On topological infinite deficiency, Michigan Math. J. 14 (1967), 365-383.

[BT] M. G. Brin and T. L. Thickstun, Open irreducible 3-manifolds which are end 1-movable, Topology 26 (1987), 211-234.

[Bo] K. Borsuk, Theory of retracts, vol. 44, PWN, Warsaw, 1967.

[Br] K. Brown, Cohomology of groups, Springer-Verlag, 1982.

[C] A. Casson, lecture at Stanford Univ., March 1990.

[Ch] T. A. Chapman, Lectures on Hilbert cube manifolds, CBMS Regional Conf. Ser. in Math., Amer. Math. Soc., Providence, RI, 1976.

[ChFe] T. A. Chapman and S. Ferry, Approximating homotopy equivalences by homeomorphisms, Amer. J. Math. 101 (1979), 583-607.

[ChS] T. A. Chapman and L. C. Siebenmann, Finding a boundary for a Hilbert cube manifold, Acta Math. 137 (1976), 171-208.

[DJ] M. W. Davis and T. Januszkiewicz, Hyperbolization of polyhedra, preprint.

[DS] J. Dydak and J. Segal, Shape theory, Lecture Notes in Math., vol. 688, Springer-Verlag, 1978.

[Dr] A. N. Dranišnikov, On a problem of P. S. Alexandrov, Mat. Sb. 135 (177) (1988), 551-557; transl. Math USSR-Sb. 63 (1989), 539-545.

[Fe] S. Ferry, Homotoping e-maps to homeomorphisms, Amer. J. Math. 101 (1979), 567-582.

[FrN] French notes: M. Coornaert, T. Delzant, and A. Papadopoulos, Géometrie et théorie des groupes: les groupes hyperboliques de Gromov, Lecture Notes in Math., vol. 1441, SpringerVerlag, 1990.

[Gr] M. Gromov, Hyperbolic groups, Essays in Group Theory (S. Gersten, ed.), MSRI publications, no. 8, Springer-Verlag, 1987.

[He] D. W. Henderson, Z-sets in ANRs, Trans. Amer. Math. Soc. 213 (1975), 205-216.

[Ja] W. Jakobsche, Approximating homotopy equivalences of surfaces by homeomorphisms, Fund. Math. 143 (1983), 1-9.

[Po] V. Poenaru, Almost convex groups, Lipschitz combing, and $\pi_{1}^{\infty}$ for universal covering spaces of closed 3-manifolds, Orsay prépublication 90-09. 
[SwN] Swiss notes: E. Ghys and P. de la Harpe (eds.), Sur les groupes hyperboliques d'après Mikhael Gromov, Birkhäuser, 1990.

[USN] U. S. notes: T. Brady, D. Cooper, T. Delzant, M. Lustig, M. Mihalik, M. Shapiro, H. Short, and A. N. Other, Notes on negatively curved groups.

[Wa] J. J. Walsh, Dimension, cohomological dimension and cell-like mappings, Shape Theory and Geometric Topology Conference, Dubrovnik, 1981 (S. Mardešić and J. Segal, eds.), Lecture Notes in Math., vol. 870, Springer-Verlag, 1981, pp. 105-118.

[Wi] R. L. Wilder, Topology of manifolds, Amer. Math. Soc. Colloq. Publ., vol. 32, Amer. Math. Soc., Providence, RI, 1949.

Department of Mathematics, University of California, Los Angeles, California 90024 